Supporting Information

\title{
From Helixes to Mesostructures: Evolution of Mesoporous Silica Shells on Single-Walled Carbon Nanotubes
}

Yao Wang, ${ }^{\dagger}$ Hao Song, ${ }^{\ddagger}$ Chengzhong $\mathrm{Yu} \stackrel{\ddagger}{\ddagger}, *$ and Hongchen $\mathrm{Gu}^{\dagger}{ }^{\dagger} *$

$\dagger$ State Key Laboratory of Oncogenes and Related Genes, School of Biomedical Engineering, Shanghai Jiao Tong University, Shanghai 200030, China

* Australian Institute for Bioengineering and Nanotechnology, The University of Queensland, Brisbane QLD 4072, Australia

\section{Corresponding Author}

*E-mails: c.yu@uq.edu.au (C.Y.); hcgu@sjtu.edu.cn (H.G.). 


\section{Supplementary Figures}

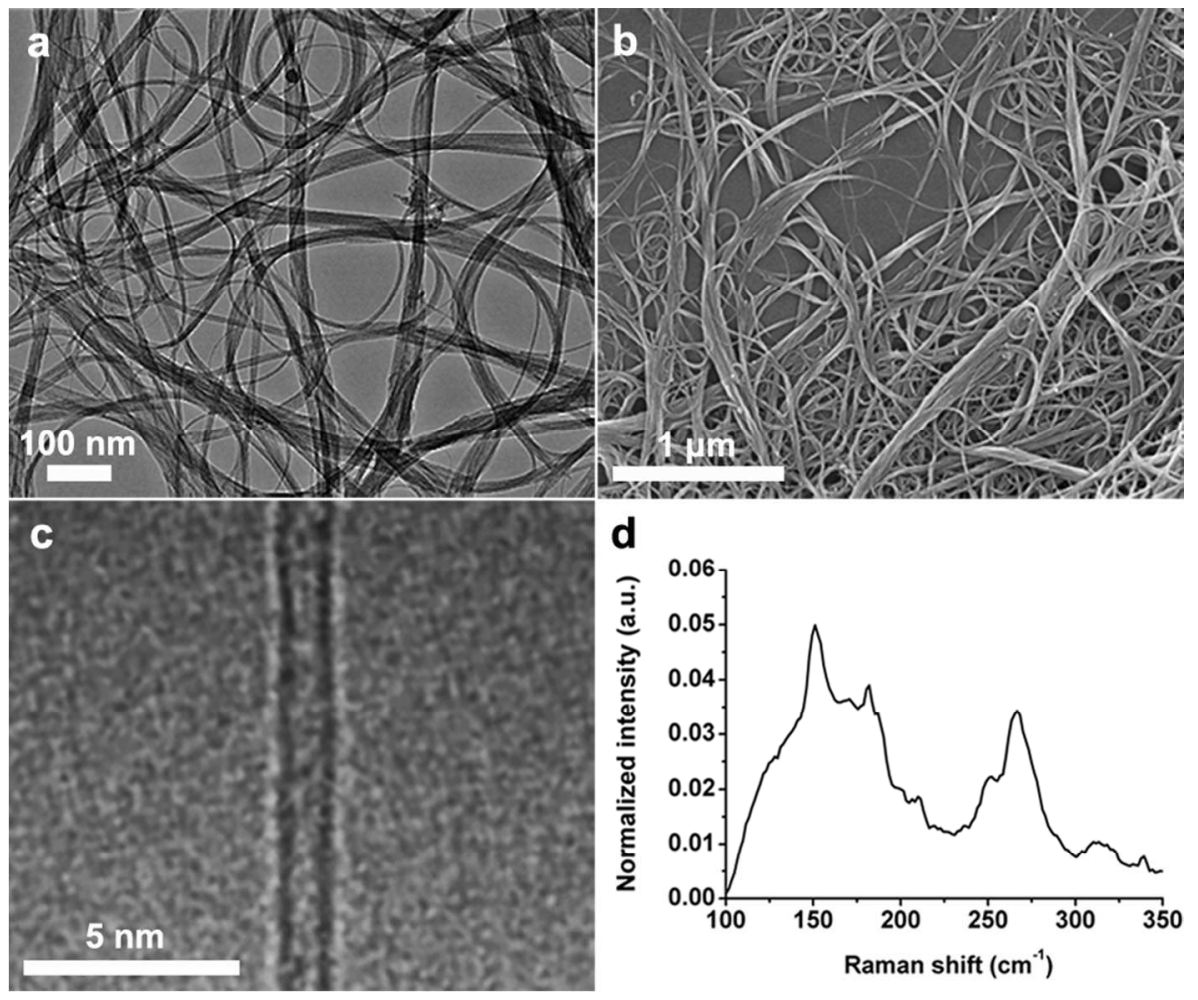

Figure S1. (a) TEM and (b) SEM images of pristine SWCNTs showing the formed bundles. (c) Representative HRTEM image of SWCNTs. (d) The RBM region $\left(100-350 \mathrm{~cm}^{-1}\right)$ from Raman spectrum of SWCNTs.

The diameter of SWCNTs $\left(\mathrm{d}_{\mathrm{t}}\right)$ can be calculated according to the following equation:

$\omega_{\mathrm{RBM}}=\alpha / \mathrm{d}_{\mathrm{t}}$,

where $\omega_{\mathrm{RBM}}$ is the frequency of the RBM, $\alpha$ is experimentally found to be $248 \mathrm{~cm}^{-1} \mathrm{~nm}$ for the silicon/silica substrate. ${ }^{1}$

The mean value of $\omega_{\mathrm{RBM}}$ is calculated to be $202.84 \mathrm{~cm}^{-1}$ from RBM (Figure S1d) by the integral method. Thus, $\mathrm{d}_{\mathrm{t}}=\alpha / \omega_{\mathrm{RBM}}=1.22 \mathrm{~nm}$. 


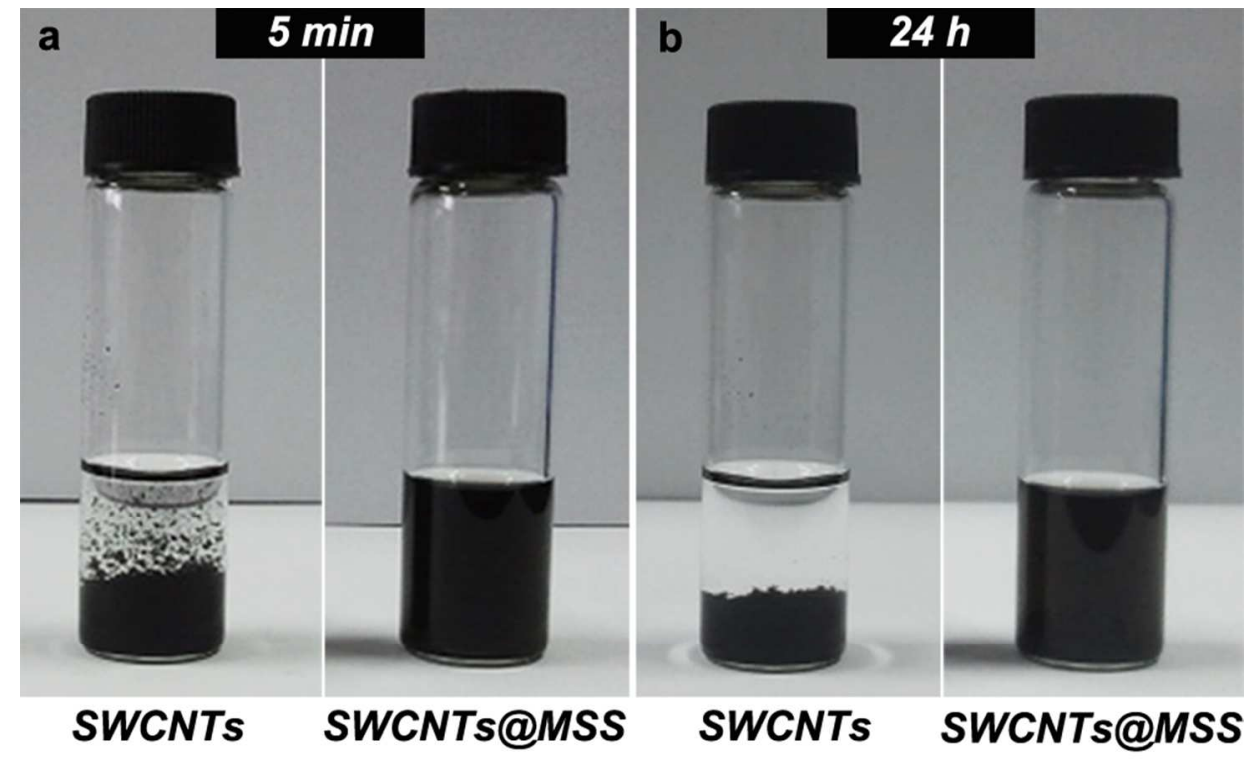

Figure S2. SWCNTs and SWCNTs@MSS in ethanol solutions: (a) 5 min after sonication, (b) $24 \mathrm{~h}$ after sonication. 

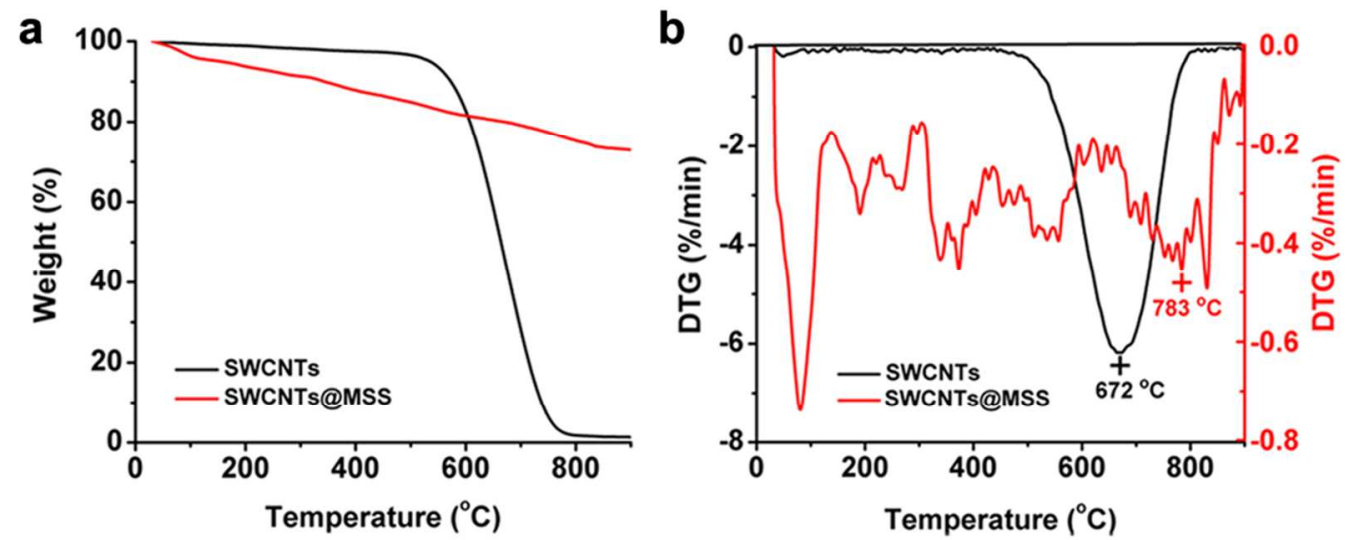

Figure S3. Thermal stability of SWCNTs and SWCNTs@MSS in air atmosphere. (a) TGA and (b) DTG curves of SWCNTs and SWCNTs@MSS. 


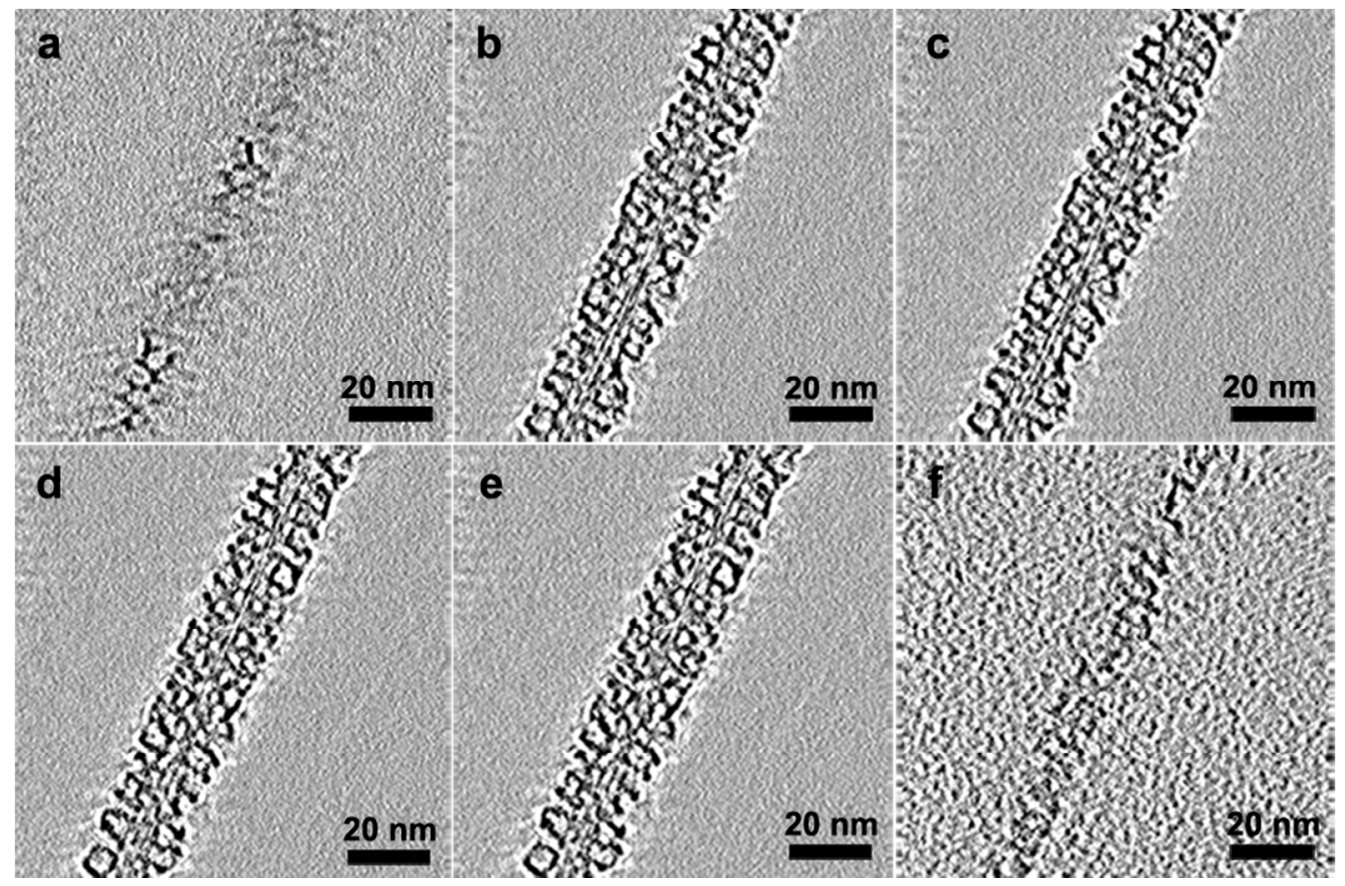

Figure S4. ET slices along the $\mathrm{z}$ axis at different positions (from top to bottom): (a) slice-1, (b) slice-34, (c) slice-35, (d) slice-37, (e) slice-38, (f) slice-72. 


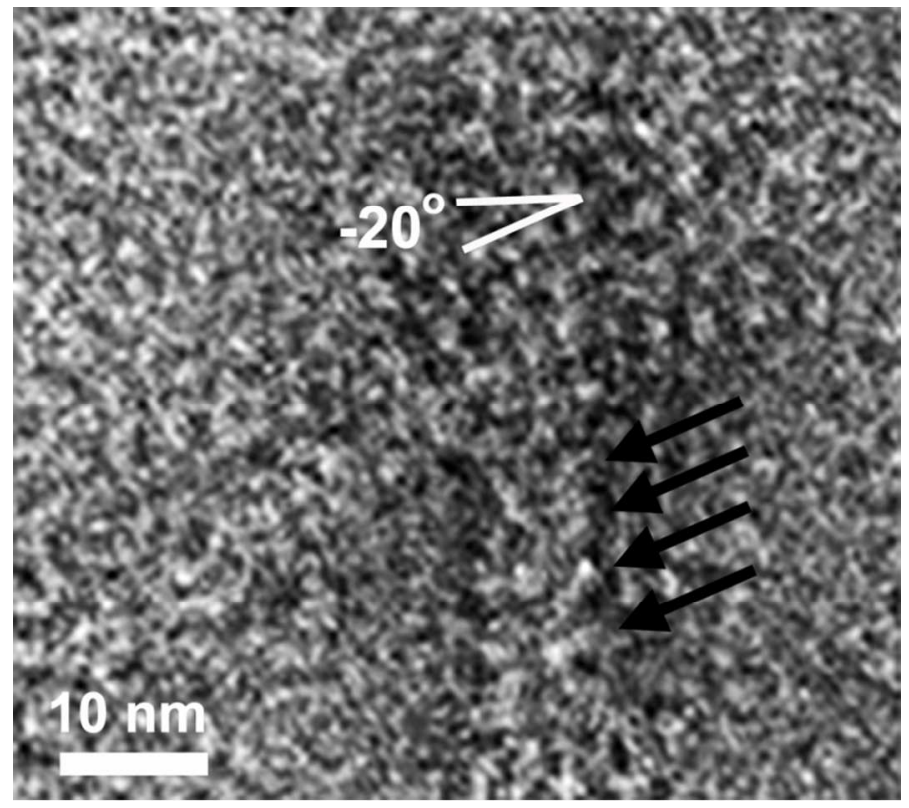

Figure S5. TEM image of SWCNTs@CTAB showing a right-handed helix with a rolled-up angle of $-20^{\circ}$. 

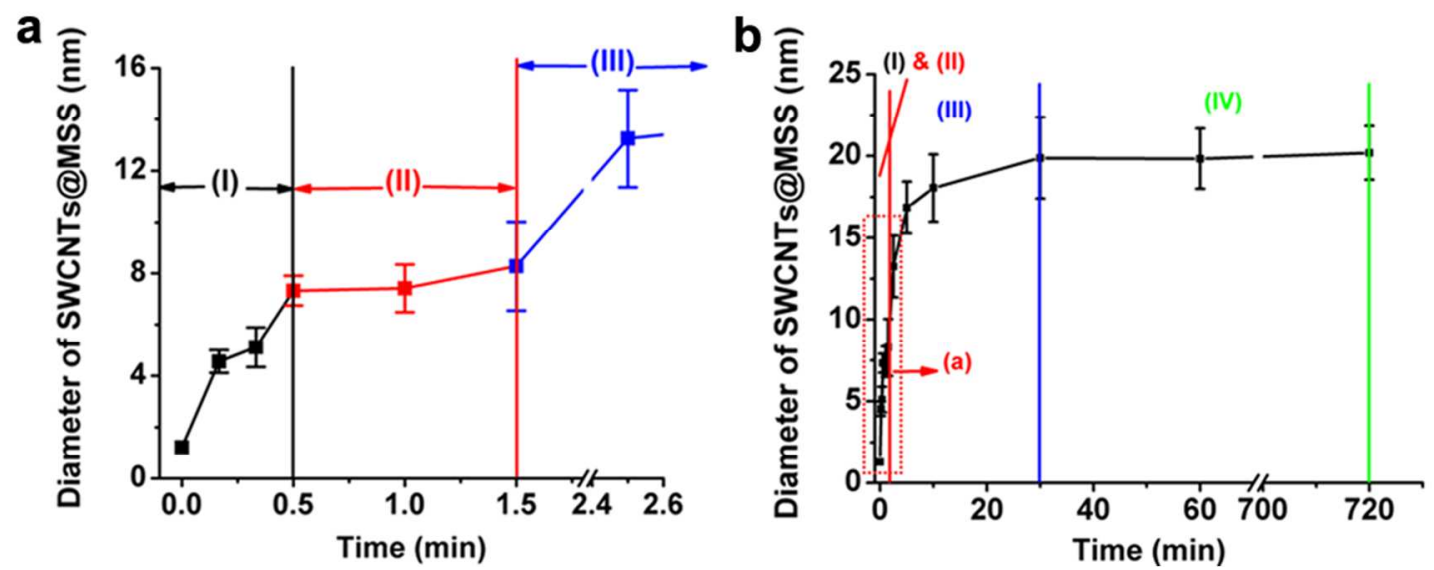

Figure S6. The diameter ${ }^{(*)}$ growth curve of SWCNTs@MSS as a function of the reaction time. Figure (a) is the enlarged region of the red square as shown in Figure (b). ${ }^{(*)}$ The outer diameter is measured for SWCNTs@MSS with helixes formed within $1.5 \mathrm{~min}$. The error bar of each data point is the standard deviation caused by the distribution of the diameter of SWCNTs@MSS. 


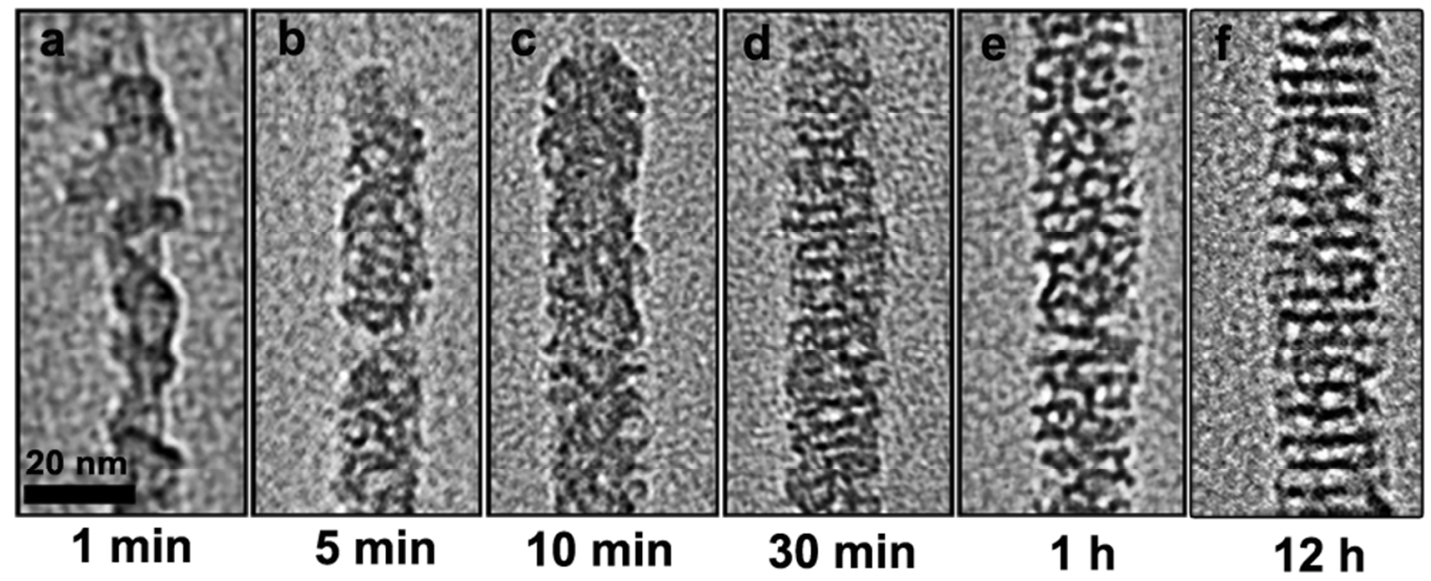

Figure S7. TEM images of SWCNTs@MSS obtained at different aging time after removal of templates: (a) $1 \mathrm{~min}$, (b) $5 \mathrm{~min}$, (c) $10 \mathrm{~min}$, (d) 30min, (e) $1 \mathrm{~h}$, and (f) $12 \mathrm{~h}$. 


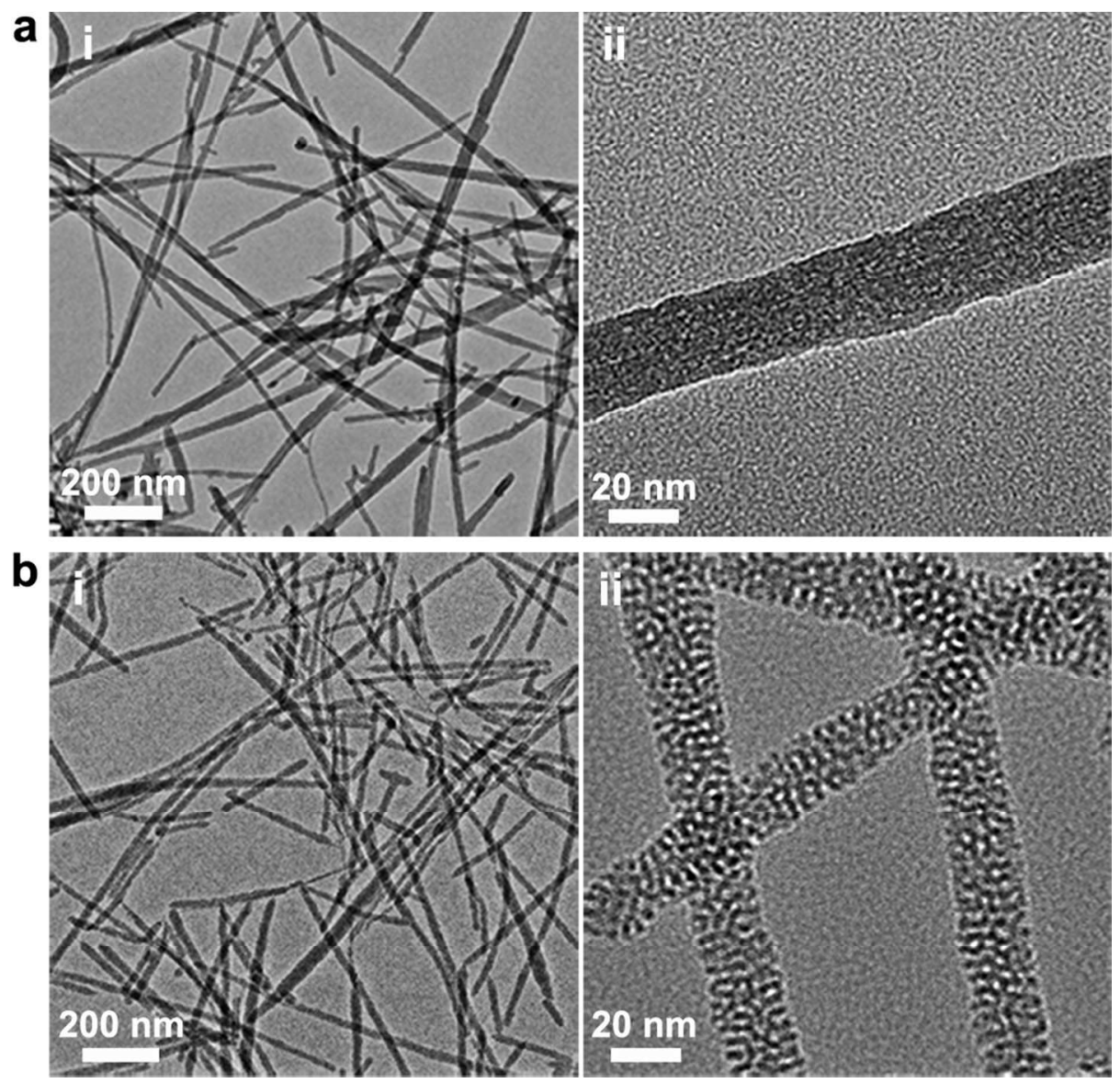

Figure S8. TEM images of SWCNTs@MSS obtained with different concentrations of CTAB: (a) 0.27 $\mathrm{mM}$, (b) $2.74 \mathrm{mM}$. (i) Low magnification and (ii) high magnification TEM images of SWCNTs@MSS. 

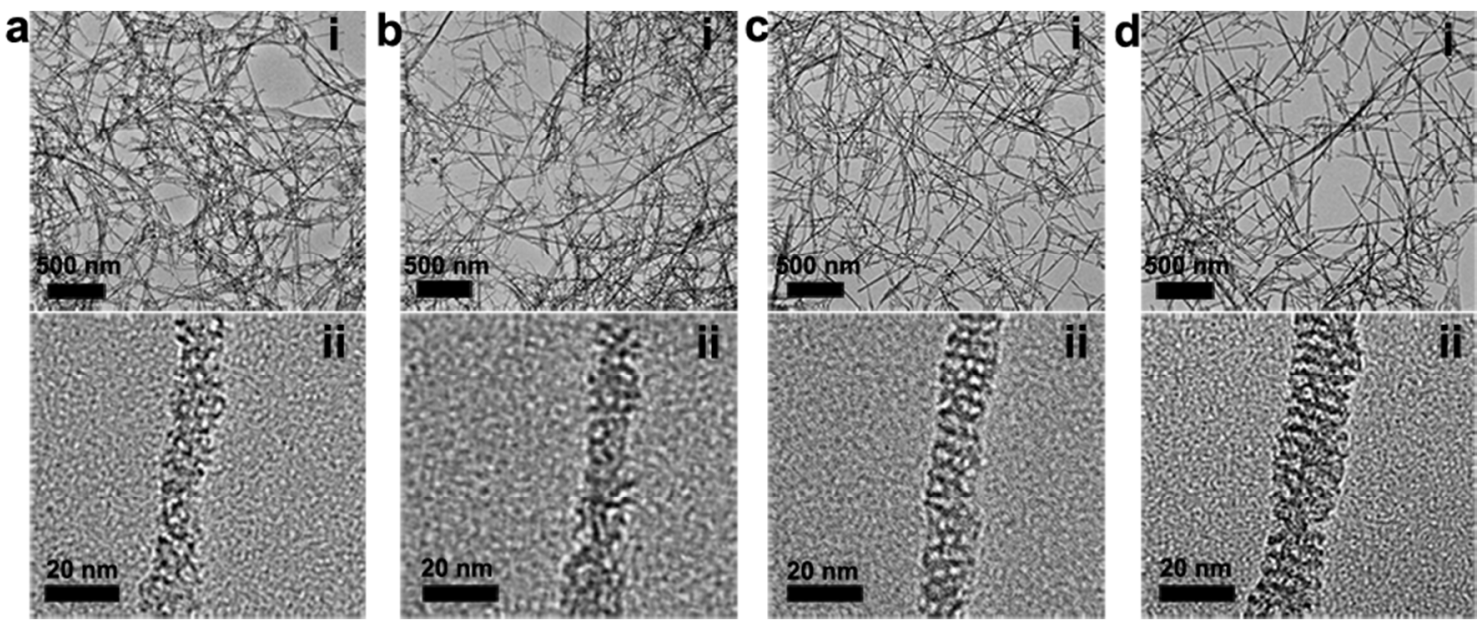

Figure S9. TEM images of SWCNTs@MSS with tunable diameters by adjusting the mass ratio (r) of added TEOS to SWCNTs: (a) $r=14.01$, (b) $r=18.68$, (c) $r=28.02$, and (d) $r=37.36$. (i) Low magnification and (ii) high magnification TEM images of SWCNTs@MSS. 

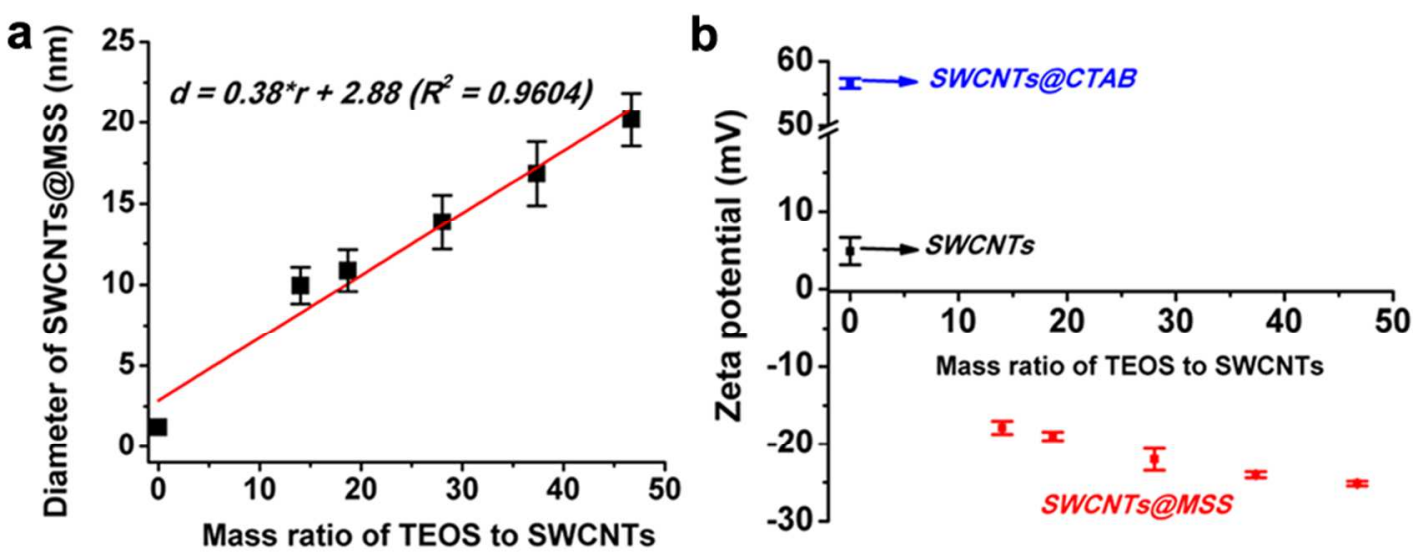

Figure S10. (a) Diameter (d) of SWCNTs@MSS as a function of TEOS-to-SWCNTs mass ratio (r). The linear fit with data points is shown as the red line. The error bar of each data point is the standard deviation caused by the distribution of the diameter of SWCNTs@MSS. (b) Zeta potential of SWCNTs, SWCNTs@CTAB, and SWCNTs@MSS as a function of TEOS-to-SWCNTs mass ratio (r). Data are presented as mean \pm standard deviation ( $\mathrm{n}=3$ independent experiments). 


\section{Reference}

(1) Dresselhaus, M.; Dresselhaus, G.; Jorio, A.; Souza Filho, A.; Saito, R. Raman Spectroscopy on Isolated Single Wall Carbon Nanotubes. Carbon 2002, 40, 2043-2061. 